\title{
Reubicación de plantas de Espeletia grandiflora (Asteraceae) como estrategia para el enriquecimiento de áreas de páramo alteradas (PNN Chingaza, Colombia)
}

\author{
Oscar Rojas-Zamora ${ }^{1}$, Jennyfer Insuasty-Torres ${ }^{1}$, Camilo de los Ángeles Cardenas ${ }^{2}$ \\ \& Orlando Vargas Ríos ${ }^{1}$ \\ 1. Grupo de Restauración Ecológica (GREUNAL) - Departamento de Biología, Facultad de Ciencias, Universidad \\ Nacional de Colombia, Carrera 30 º45-03 edificio 421, oficina 149 Bogotá, Colombia; oarojasz@unal.edu.co, jin- \\ suastyt@unal.edu.co,jovargasr@unal.edu.co \\ 2. Universidad Antonio Nariño; asesor.investigaciones@uan.edu.co
}

Recibido 30-IX-2011. Corregido 30-VI-2012. Aceptado 23-VII-2012.

\begin{abstract}
Relocation of Espeletia grandiflora (Asteraceae) plants as a strategy for enrichment of disturbed paramo areas (PNN Chingaza, Colombia). Ecological restoration of the Andean paramos faces several ecological barriers mainly at the phase of dispersal and establishment of native species. With the aim to contribute to the enrichment of degraded areas, different strategies have to be developed to overcome those barriers. In this work we studied the response of individuals of Espeletia grandiflora (Asteraceae) to the relocation as a strategy for ecological restoration programs. We also evaluated the effect of size of relocated individuals on their survival and development. The work was carried out in an experimental plot at $3424 \mathrm{~m}$ altitude in the sector "Lagunas de Siecha" of Chingaza National Park, Colombia. We relocated 200 plants that belonged to three different size classes: 5,10 and $15 \mathrm{~cm}$ of initial height. The following variables were registered: survival, plant height, number of living leaves and stem diameter of each individual. We also evaluated the differences between individuals in survival and development. In terms of survival the most efficient size classes corresponded to $15 \mathrm{~cm}$ high; the survival was $85 \%$ after two years. The relative growth rates for height and stem diameter decreases with the increase in size, but the absolute increase in height did not show significant differences between the three sizes tested. Since the stem diameter was found the strongest survival predictor after two years of relocation activities, we suggest its use as a criterion for selection of relocation individuals. The relocation of individuals of $E$. grandiflora had a positive side effect, carrying other species that may contribute to the enrichment and restoration of degraded areas. Among these, we found species of the genus Hypericum, as well as Arcytophyllum nitidum and Calamagrostis effusa, which should be evaluated in terms of survival and development for the subsequent implementation of the relocation strategy. In this study we verified the successful relocation of individuals of $E$. grandiflora as a strategy for enrichment of paramos, and provided values of survival and growth, which should be useful for planning and predicting with greater certainty the success of restoration programs in the paramo. Rev. Biol. Trop. 61 (1): 363-376. Epub 2013 March 01.
\end{abstract}

Key words: ecological restoration, transplants, survival, relative growth rate, caulescent rosette.

A pesar de las difíciles condiciones ambientales, como son las bajas temperaturas y la alta humedad, la presencia humana en la alta montaña tropical andina se remonta a cientos de años atrás (Ellenberg 1979, Molinillo \& Monasterio 1997, Sarmiento 2000), y desde entonces se ha dado una constante transformación en los ecosistemas. Uno de los principales usos de la tierra y causa de destrucción de los ecosistemas de alta montaña, como son los páramos, han sido las actividades agrícolas y la creación de pastizales para la crianza de ganado (Molinillo \& Monasterio 1997, 2002, Vargas et al. 2002). Estas actividades productivas, se acompañan de prácticas como la quema de la vegetación antes de la siembra o de la 
introducción del ganado, lo que ha generado un régimen de disturbios responsables de la transformación de la vegetación a parches con diferentes grados de alteración. Estos regímenes de disturbio modifican las condiciones ambientales, la composición florística, la diversidad, estructuras poblacionales de plantas y animales, y transforman la estructura de las comunidades vegetales reduciendo de esta manera el potencial natural de regeneración del ecosistema (Sturm \& Rangel 1985, Molinillo 1992, Verweij \& Kok 1992, Hofstede 1995, Hofstede et al. 1995, Vargas et al. 2002, Vargas \& Pedraza 2004).

Las áreas de páramo alteradas por pastoreo presentan especies nativas y exóticas altamente competitivas, principalmente pastos y plantas rasantes como Lachemilla orbiculata, Rumex acetosella, Anthoxanthum odoratum, que limitan la germinación de otras especies propias del páramo (Vargas et al. 2002, Jaimes \& Sarmiento 2002). Los pastos pueden permanecer dominantes por décadas en los campos abandonados, dificultando la implantación, establecimiento y reclutamiento de individuos de especies nativas, tanto en páramo (Sarmiento et al. 2003), como en otros ecosistemas (Aide \& Cavelier 1994, Slocum 2000, Zimmerman et al. 2000). Esta condición lleva a lo que Grime (2001) define como una comunidad pro-clímax, causada por disturbios recurrentes y continuos, donde predomina el estrato herbáceo dominado por especies introducidas, y que algunos autores describen este fenómeno como "sucesión detenida" (Sarmiento 1997, Holl 2002, Meli 2003). Estas alteraciones pueden modificar la estructura, composición y funcionalidad del ecosistema poniendo en peligro la permanencia de muchas especies, así como la prestación de servicios ambientales como la producción y regulación del agua (Buytaert et al. 2006, Suarez 2008); en consecuencia, surge la necesidad de dar respuestas a la problemática de alteración de estas zonas y tomar acciones para reparar los efectos no deseados de la intervención humana.

El trasplante o reubicación de individuos ha sido ampliamente usado para la adición de especies en procesos de restauración, y puede ser una estrategia eficaz para superar las etapas vulnerables de germinación y reclutamiento en sitios con condiciones físicas o ambientales extremas (May et al. 1982, Urbanska 1994, Fattorini 2001, Orozco 2007, Trujillo-Ortiz 2007, García-Orth \& Martínez-Ramos 2011, Wallin et al. 2009). Autores como Sarmiento et al. (2003) sugieren la siembra de especies de etapas intermedias y tardías de la sucesión como una manera de acelerar la restauración en áreas de páramo. Adicionalmente, la estrategia pueden aumentar la lluvia de semillas al generar perchas vivas (Holl 2002), generar sombra que puede limitar el establecimiento y crecimiento de los pastos y otras plantas invasoras (Guevara et al. 1986, Díaz-Espinosa \& Vargas 2009), aumentar la producción de hojarasca que puede activar los ciclos de nutrientes en el suelo (Vázquez-Yanes \& Orozco-Segovia 1993), por lo que facilita los procesos de sucesión (Aide et al. 1995, Nepstad et al. 1996, Parrotta \& Knowles 1999). Sin embargo, son varios los aspectos que se deben conocer y tener en cuenta para llevar a cabo la reubicación de individuos de una manera eficaz. En particular, se requiere conocimiento sobre la autoecología y el papel dentro de la comunidad de las especies vegetales que presentan mayor abundancia, definen las comunidades o que son de importancia para la dinámica del ecosistema, así mismo, se requiere conocer su respuesta a las condiciones micro ambientales generadas por los disturbios $\mathrm{y}$ ante las cuales algunas especies nativas no pueden permanecer (Vargas et al. 2002).

Las especies del género Espeletia, conocidas comúnmente como frailejones, son plantas con varias adaptaciones morfológicas y fisiológicas al clima extremo del páramo (Monasterio \& Sarmiento 1991), tienen una forma de crecimiento en roseta protegiendo el punto de vegetativo (Smith 1974), algunas especies mantienen las hojas marcescentes para aislar el tronco de bajas temperaturas y retener nutrientes (Smith 1979, Goldstein \& Meinzer 1983), poseen una médula al interior del tronco que permite retener agua (Azócar \& Rada 2006), desarrollan hojas suculentas y pubescentes, 
presentan alta resistencia a los rayos UV-B (Baruch \& Smith 1979, Meinzer \& Goldstein 1985), e inclusive generan metabolitos que frente a bajas temperaturas inhiben la congelación del agua en el citoplasma (Goldstein et al. 1984, García-Varela \& Rada 2003). Estas características, junto al hecho de ser las plantas más conspicuas de los páramos andinos, hacen de las especies de este género plantas de interés en los procesos de restauración ecológica de los páramos andinos.

Espeletia grandiflora Bonpl. es una especie de frailejón característica de los páramos de suelos bien drenados de la cordillera Oriental de Colombia, y define la comunidad predominante de estos páramos (Lozano \& Schnetter 1976, Rangel-Ch \& Ariza-N 2000); E. grandiflora solo es encontrada como adulto en los páramos con tiempos de regeneración de entre 15 y 20 años (Jaimes \& Sarmiento 2002), por lo que su reintroducción puede ser importante para adelantar procesos de restauración. Hasta ahora no existen estudios que evalúen el éxito de la estrategia de reubicación de plantas en pastizales de páramo para propiciar un re-direccionamiento de la sucesión en estas áreas, así como la eficiencia y eficacia e implicaciones ecológicas asociadas al tamaño de los individuos reubicados. En este estudio se evaluó el efecto del tamaño de las plantas de E. grandiflora reubicadas sobre el desempeño (supervivencia y crecimiento) de las mismas. El pastizal donde se desarrolló el estudio corresponde a un área que fue objeto de actividades de agricultura y pastoreo de ganado vacuno hasta el año 2000 aproximadamente; estas actividades productivas se encontraban acompañadas de prácticas de remoción de la vegetación nativa por medio de corte, quemas periódicas y arado del suelo.

\section{MATERIALES Y MÉTODOS}

Área de estudio: El estudio se realizó entre mayo 2008 y abril 2010 en un área de páramo pastoreada en el sector "Lagunas de Siecha" en el noroccidente del Parque Nacional Natural Chingaza, Colombia, ubicado a los 446' $10^{\prime \prime} \mathrm{N}-73^{\circ} 51^{\prime} 53.9^{\prime}$ " W y $3424 \mathrm{~m}$ de altitud en la cordillera Oriental de los Andes colombianos. Las precipitaciones anuales ubican a Chingaza como un páramo húmedo, el régimen de lluvias es unimodal con una sola época seca de diciembre a febrero, y el periodo de mayores precipitaciones hacia junio y julio. La pluviosidad promedio anual del sector es de $1200 \mathrm{~mm}$. Las temperaturas medias anuales del Parque oscilan entre 6.7 y $7.9^{\circ} \mathrm{C}$, y pueden presentar grandes variaciones durante el día. La distribución de temperaturas durante el año es antagónica al patrón de distribución de la precipitación: a altas pluviosidades corresponden bajas temperaturas y viceversa (Vargas \& Pedraza 2004).

El área de estudio corresponde a pastizal de páramo con historia reciente de intervención humana por actividades ganaderas y agrícolas, el área se encuentra en descanso desde hace ocho años, y su fisonomía es de un pastizal dominado por Anthoxantum odoratum, con menor presencia de Poa annua y Holcus lanatus, y sin presencia de E. grandiflora. El área de estudio se encuentra aledaña a páramos conservados que se encuentran dominados por $E$. grandiflora, sin embargo el área se cercó para evitar la entrada de ganado.

Especie de estudio: E. grandiflora es una planta monoica, perteneciente a la familia de las compuestas (Asteraceae) y común de los páramos con suelos bien drenados de la cordillera Oriental de Colombia. Es una roseta que alcanza una altura máxima aproximada de dos metros, acumula hojas marcescentes en el tallo y presenta abundante indumento sobre las hojas, el eje de las inflorescencias y el pedúnculo de las flores. El eje de la inflorescencia compuesta es mucho más largo que la roseta y presenta indumento generalmente amarillo. Las inflorescencias simples (capítulos) están conformadas por un gran número de flores simples femeninas (flósculos) y masculinas (lígulas). Además de ser una de las plantas más conspicuas de los páramos colombianos, E. grandiflora presenta gran importancia en el funcionamiento del ecosistema debido a su 
productividad (Hernández \& Murcia 1995) y a que alberga una variada y abundante fauna entre las hojas marcescentes que mantiene y en sus capítulos (Sendoya \& Bonilla 2005, Fagua \& González 2007).

Reubicación de plantas: Las plantas de E. grandiflora se extrajeron de zonas de páramo sin alteraciones con alta densidad de individuos; estas áreas se encuentran a una distancia no menor de $1 \mathrm{~km}$ del área de estudio, dominado por la comunidad de E. grandifloraCalamagrostis effusa, con algunos arbustos de Arcytophyllum nitidum, Hypericum goyanesii y Pentacalia ledifolia; la extracción de las plantas se realizó en mayo 2008, causando el menor impacto a las raíces al ser retiradas con una porción de suelo de $400 \mathrm{~cm}^{2}$ alrededor de la planta y de $20 \mathrm{~cm}$ de profundidad (Fig. 1), por lo que en ocasiones llevaron consigo individuos de otras plantas de la comunidad de páramo. Se establecieron tres categorías para evaluar el efecto del tamaño sobre el desempeño de las plantas reubicadas: La categoría "pequeño" se encuentra entre 3 y $7 \mathrm{~cm}$ de altura, la categoría "medio" entre 8 y $12 \mathrm{~cm}$ de altura, y la categoría "grande" entre 13 y $17 \mathrm{~cm}$ de altura. Se extrajeron 200 plantas de cada una de las categorías de tamaño, las cuales se plantaron en ocho parcelas de $2 \times 2 \mathrm{~m}$ donde previamente se eliminó la biomasa epigea para eliminar de manera temporal la competencia que puede ocasionar los pastos exóticos. En cada parcela, se plantaron 25 plantas de único tamaño, distribuidas en filas de cinco plantas cada una y separadas entre sí por una distanciada de $50 \mathrm{~cm}$. Con el fin de evaluar el desempeño de las plantas se tomaron mediciones a los $3.1,9.5,13.1,18.7$ y 24 meses luego de la reubicación. A cada individuo se le midió la altura como la distancia desde la base del tallo hasta el ápice de la hoja de mayor tamaño, se midió el diámetro del tallo (DT) y se contó el número de hojas vivas. En cada medición se estimó la supervivencia como el porcentaje de individuos vivos en cada parcela, el incremento en altura como la diferencia de altura entre el intervalo de tiempo, y en el diámetro del tallo como la diferencia en el diámetro del tallo en el tiempo. Para cada uno de los individuos, se calculó la tasa de crecimiento relativo en altura medida en centímetros $\left(\mathrm{TCR}_{\mathrm{A}}\right)$ y la tasa de crecimiento relativo en diámetro del tallo medida en milímetros $\left(\mathrm{TCR}_{\mathrm{DT}}\right)$, la fórmula usada fue $\mathrm{TCR}=[\ln (\operatorname{tamaño}$ final $)-\ln (\operatorname{tamaño}$ inicial)]/tiempo (Fisher 1921, Hunt 1990); la tasa de renovación de hojas se calculó como $\mathrm{TRH}=$ (número final de hojas-número inicial de hojas)/tiempo.

La probabilidad de supervivencia de las plantas se calculó a los 3.1, 9.5, 13.1, 18.7 y 24 meses luego de la reubicación. En el caso
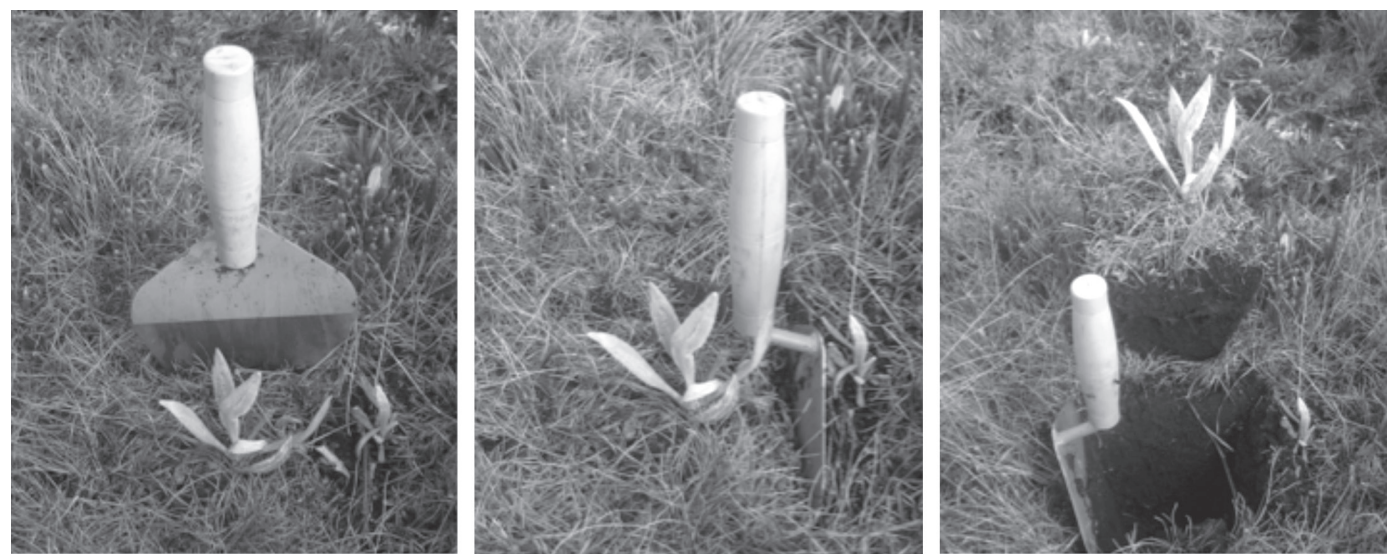

Fig. 1. Extracción de plantas jóvenes de E. grandiflora para ser reubicados en pastizales de páramo.

Fig. 1. Extraction of E. grandiflora saplings to be relocated in paramo pastures. 
del tiempo se usó como nivel de referencia la supervivencia a los 3.1 meses luego de la reubicación. Se usó el modelo probabilístico Binomial (viva/muerta), por lo que se aplicó un modelo de regresión logística considerando la supervivencia como variable de respuesta, y el tiempo y categoría de tamaño inicial como factores. Se estimaron los parámetros del modelo y se usó el estadístico de chi $^{2}$ de Wald, con el método de máxima verosimilitud, para probar el efecto de los factores sobre la supervivencia de las plántulas. Con el fin de determinar cuáles de las variables entre altura, diámetro del tallo y número de hojas, son las más adecuadas para predecir la supervivencia 24 meses después de la reubicación, se realizó un análisis de regresión logística paso a paso hacia atrás mediante el método de Wald, usando como variable respuesta la supervivencia después de 24 meses, y la altura, diámetro del tallo y el número de hojas en el momento de su extracción.

El crecimiento de las plantas fue analizado a través de modelos lineales generalizados (MLG), con un factor inter-sujetos (tamaño, con tres niveles) y un factor intra-sujetos (Tiempo, con cinco niveles). El número de hojas vivas se analizó con un modelo probabilístico de Poisson y una función de enlace Log; el incremento en altura y diámetro del tallo con un modelo probabilístico Normal con una función de enlace identidad. Las diferencias entre los tres niveles del factor Tamaño y entre los cinco niveles intra-sujetos (Tiempo a 3.1, 9.5, $13.1,18.7$ y 24 meses) se analizaron usando comparaciones pareadas de Bonferroni.

Para evaluar las diferencias en crecimiento total entre las categorías de tamaño se usaron pruebas no paramétricas de Kruskal-Wallis, con las variables dependientes altura, diámetro del tallo y número de hojas finales de las plantas que sobrevivieron hasta la última medición. Las medias y los valores de error estándar se calcularon para cada una de las categorías de tamaño inicial. Los análisis se realizaron usando el paquete estadístico SPSS 19 para Windows (SPSS, Inc.) y Statgraphics Centurion XV (StatPoint, Inc.).

\section{RESULTADOS}

En general, el $74.62 \%(\mathrm{ES}=2.6 \%)$ de todos los trasplantes $(\mathrm{n}=600)$ se encontraban vivos 24 meses después de la reubicación. Las plantas reubicadas de diferentes categorías de tamaño presentaron diferencias en su supervivencia, siendo esta mayor en las plantas de tamaño Grande (Cuadro 1, Fig. 2). El tiempo también afectó significativamente la supervivencia,

CUADRO 1

Regresión logística mediante un modelo lineal generalizado para estimar la supervivencia de las plantas jóvenes de E. grandiflora reubicados en pastizales de páramo

TABLE 1

Logistic regression using a generalized linear model to estimate survival of E. grandiflora saplings relocated in paramo pastures

\begin{tabular}{lcccccccc} 
Factores y sus niveles & $\beta$ & ES & Wald & g.l. & p & Exp $(\beta)$ & Intervalos de confianza (95\%) \\
Tamaño & & & 26.755 & 2 & $<0.01$ & & & Superior \\
Tamaño (pequeño) & 3.356 & 0.268 & 156.776 & 1 & $<0.01$ & 28.681 & 2.831 & 3.882 \\
Tamaño (medio) & 3.748 & 0.273 & 188.845 & 1 & $<0.01$ & 42.446 & 3.214 & 4.283 \\
Tamaño (grande) & 4.002 & 0.276 & 209.862 & 1 & $<0.01$ & 54.697 & 3.460 & 4.543 \\
Tiempo & & & 104.220 & 4 & $<0.01$ & & & -1.189 \\
Tiempo (9.5 meses) & -1.755 & 0.289 & 36.877 & 1 & $<0.01$ & 0.173 & -2.321 & -1.520 \\
Tiempo (13.7 meses) & -2.076 & 0.284 & 53.516 & 1 & $<0.01$ & 0.125 & -2.632 & -1.760 \\
Tiempo (18.1 meses) & -2.310 & 0.281 & 67.627 & 1 & $<0.01$ & 0.099 & -2.861 & -2.062 \\
Tiempo (24 meses) & -2.607 & 0.278 & 87.797 & 1 & $<0.01$ & 0.074 & -3.152 & \\
\hline
\end{tabular}




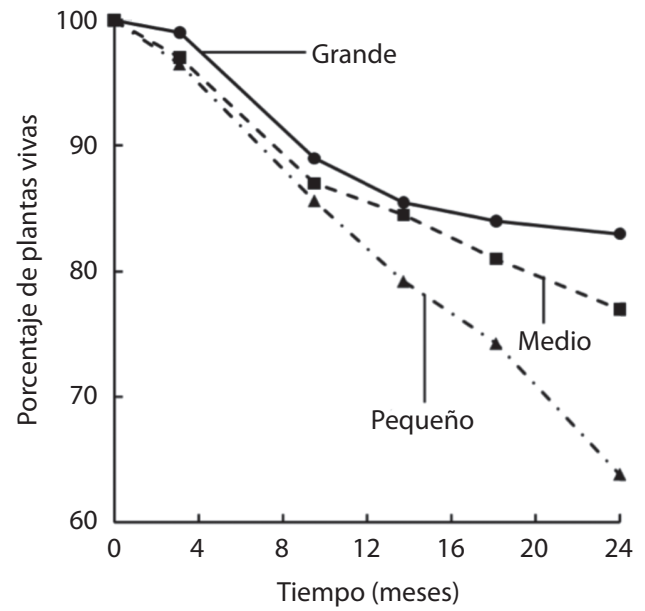

Fig. 2. Efecto de la categoría de tamaño inicial sobre el comportamiento de la supervivencia de plantas jóvenes de E. grandiflora reubicados en pastizales de páramo a lo largo de 24 meses de estudio.

Fig. 2. Effect of initial size category on the behavior of survival throughout 24 months study of E. grandiflora saplings relocated in paramo pastures.

estando entre un $99-96 \%$ a los 3.1 meses hasta disminuir a un 83-63\% a los 24 meses (Fig. 2). A partir del análisis de regresión logística se tiene que la variable que mejor permite predecir la probabilidad de supervivencia dos años después de la reubicación es el diámetro inicial del tallo medido en centímetros (Cuadro 2). La probabilidad de supervivencia puede ser estimada como Supervivencia $=\mathrm{e}^{\left(0.178+1.25^{*} \mathrm{DT} \text { inicial }\right) /}$ $\left(1+\mathrm{e}^{(0.178+1.25 * \mathrm{DT} \text { inicial })}\right)$

La categoría de tamaño Grande siempre presentó los mayores niveles de supervivencia, seguido de la categoría Medio y finalmente las plantas de categoría Pequeño. En general, la supervivencia de las tres categorías se comporta de manera similar hasta un año después de la siembra; sin embargo, las plantas de la categoría Pequeño presentan un incremento en la mortalidad durante el segundo año posterior a la reubicación (Fig. 2). Para las tres categorías de tamaño la mayor mortalidad se presentó entre los 3.1 y 9.5 meses y los 18.1 y 24 meses después de la reubicación, periodos de tiempo que incluyen los meses más secos en el área de estudio (diciembre a febrero).

Luego de 24 meses de estudio, las plantas tanto de la categoría Pequeños como Medios crecieron lo suficiente para pasar a la categoría de tamaño siguiente (Fig. 3A). Las plantas de categoría Pequeña presentaron un incremento anual en altura promedio de 2.87; desv. estándar $1.64 \mathrm{~cm} ; \mathrm{n}=200$, mientras que para los individuos Medios fue de $3.0 ; 2.01 \mathrm{~cm}$, y para la categoría Grande es de $3.2 ; 2.37 \mathrm{~cm}$. Para la variable incremento en altura el factor Tiempo fue el único que presentó efectos significativos (Cuadro 3), donde el mayor crecimiento se presentó entre los 9.5 y 13.1 meses luego de la reubicación (Fig. 3A). La variable incremento en el diámetro del tallo solo se presentó efectos significativos para los factores principales Tamaño y Tiempo, pero no para su interacción (Cuadro 3). El incremento en diámetro del tallo presentado por los individuos de categoría Grande fue mayor y difiere significativamente del incremento presentado por las otras dos categorías (Bonferroni, $\mathrm{p}<0.05$ ); donde el mayor crecimiento se presentó, de manera similar a lo ocurrido con el incremento en altura, entre los 9.5 y 13.1 meses luego de la reubicación, periodo que corresponde a los meses

CUADRO 2

Estimación de la supervivencia de las plantas jóvenes de E. grandiflora luego de 24 meses de ser reubicados en pastizales de páramo

TABLE 2

Estimate of survival of E. grandiflora saplings after 24 months of being relocated in paramo pastures

\begin{tabular}{lcccccc}
\multicolumn{1}{c}{ Factor } & $\beta$ & ES & Wald & g.l. & p & $\exp (\beta)$ \\
Diámetro inicial del tallo & 1.252 & 0.312 & 16.105 & 1 & $<0.01$ & 3.498 \\
Constante & 0.178 & 0.235 & 0.577 & 1 & 0.447 & 1.195 \\
\hline
\end{tabular}


A
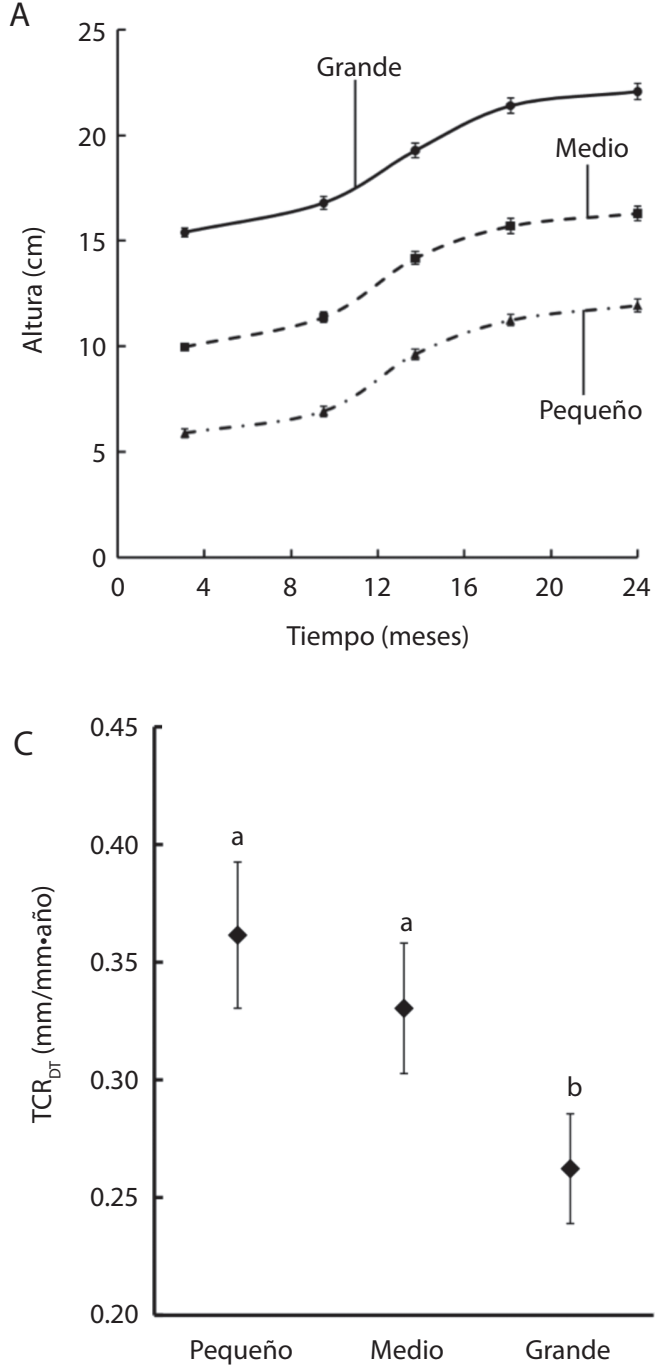

B
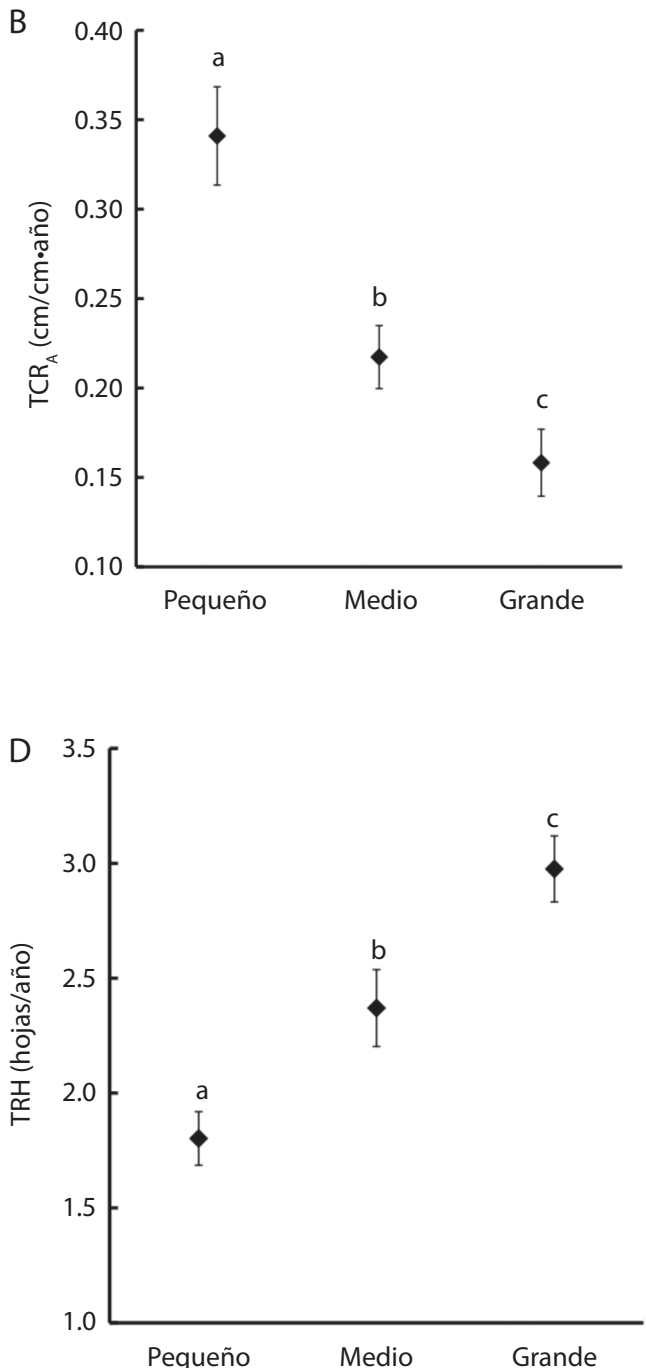

Fig. 3. Efecto del tamaño de los individuos sobre las tasas de crecimiento de las plantas de Espeletia grandiflora reubicadas en pastizales de páramo luego de 24 meses de estudio. (A) Trayectoria del crecimiento en altura. (B) tasa de crecimiento anual relativa en altura $\left(\mathrm{TCR}_{\mathrm{A}}\right)$. $(\mathrm{C})$ tasa de crecimiento anual relativa en diámetro del tallo $\left(\mathrm{TCR}_{\mathrm{DT}}\right)$, y $(\mathrm{D})$ tasa de renovación anual de hojas (TRH). Las líneas verticales indican el error estándar. Letras diferentes indican diferencias significativas $(\mathrm{p}<0.05)$.

Fig. 3. Effect of individual's size on the growth rates of E. grandiflora saplings relocated in paramo pastures after 24 months of study. (A) Height growth trajectory. (B) Relative growth rate in height $\left(\mathrm{TCR}_{\mathrm{A}}\right)$. (C) Relative growth rate in stem diameter $\left(\mathrm{TCR}_{\mathrm{DT}}\right)$ and $(\mathrm{D})$ Annual renewal rate of leaves $(\mathrm{TRH})$. The vertical lines indicate the standard error. Different letters indicate significant differences $(\mathrm{p}<0.05)$.

posteriores a la época seca (marzo-junio). Para la variable Número de hojas, los factores Tamaño, Tiempo y la interacción entre los dos fue significativa (Cuadro 3). El mayor número de hojas en las tres categorías de Tamaño se dio a los 18.1 meses de la reubicación, y en todos los tiempos se presentaron diferencias entre las categorías de tamaño (Bonferroni, $\mathrm{p}<0.05$ ). Para la tasa de crecimiento relativa mensual en altura $\left(\mathrm{TCR}_{\mathrm{A}}\right)$, tanto el factor Tiempo, el 


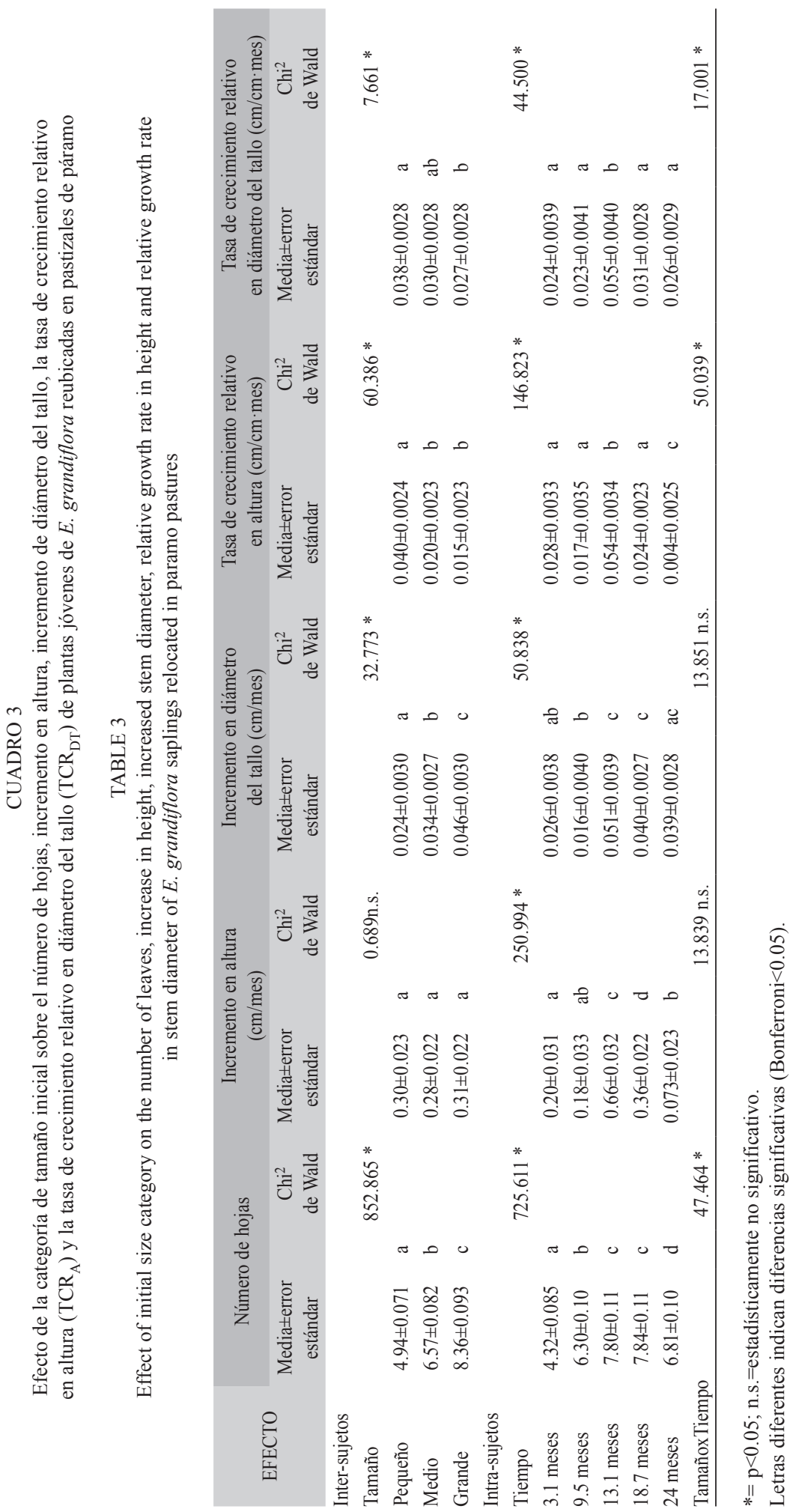


Tamaño, como su interacción tuvieron efectos significativos (Cuadro 3), donde el mayor crecimiento se presentó para los individuos de tamaño Grande y entre los 9.5 y 13.1 meses luego de la reubicación. De igual manera, para la tasa de crecimiento relativa mensual del diámetro del tallo $\left(\mathrm{TCR}_{\mathrm{DT}}\right)$ se presentaron efectos significativos para todos los factores principales, Tamaño y Tiempo, y para su interacción (Cuadro 3); siguiendo el mismo comportamiento registrado para $\mathrm{TCR}_{\mathrm{A}}$.

La categoría de tamaño de los individuos reubicados presentó un efecto sobre las TCR anuales, siendo estas mayor en los individuos de categoría Pequeño (Fig. 3B y 3C), la $\mathrm{TCR}_{\mathrm{A}}$ en la categoría Pequeño $(0.360 ; 0.029 \mathrm{~cm} /$

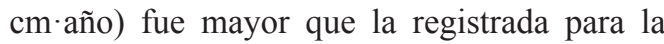
categoría Medio $(0.229 ; 0.018 \mathrm{~cm} / \mathrm{cm} \cdot a n ̃ o)$ y Grande $(0.166 ; 0.019 \mathrm{~cm} / \mathrm{cm} \cdot$ año $)(\mathrm{H}=35.2815$, $\mathrm{p}<0.001)$; así mismo, la $\mathrm{TCR}_{\mathrm{DT}}$ de la catego-

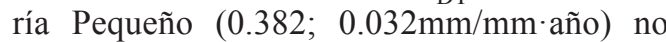
presentó diferencias a la obtenida por la categoría Medio $(0.349 ; 0.029 \mathrm{~mm} / \mathrm{mm} \cdot a n ̃ o)$ y fue $37 \%$ mayor que la registrada para la categoría Grande $(0.277 ; 0.024 \mathrm{~mm} / \mathrm{mm} \cdot a n ̃ o)$ que tuvo la $\mathrm{TCR}_{\mathrm{DT}}$ más baja $(\mathrm{H}=8.3176, \mathrm{p}=0.0156)$. La tasa anual de renovación de hojas TRH presentó un comportamiento contrario a lo obtenido para $\mathrm{TCR}_{\mathrm{A}}$ y $\mathrm{TCR}_{\mathrm{DT}}$ (Fig. 3D) siendo mayor en la categoría Grande (3.149; 0.152 hojas/año) respecto a Pequeño $(1.907 ; 0.123$ hojas/año) y Medio (2.508; 0.177 hojas/año) $(\mathrm{H}=31.4605, \mathrm{p}<0.001)$.

\section{DISCUSIÓN}

La reubicación o trasplante de individuos para el enriquecimiento de áreas degradadas con miras a la restauración ecológica es una estrategia que ha sido ampliamente probada en diversos ecosistemas y ambientes desde hace varias décadas, y en general puede considerarse una estrategia eficaz para ecosistemas donde los procesos de regeneración son lentos debido a la baja productividad primaria neta, las bajas temperaturas, las altas variaciones de temperatura entre el día y la noche, y los suelos ácidos, tal como ocurre en la alta montaña tropical
(Sturm \& Rangel 1985, Rangel \& Sturm 1995). Brown \& Lugo (1994) plantean cinco conjuntos de estrategias para llevar a cabo la rehabilitación de áreas tropicales degradadas, entre las cuales se encuentra un conjunto de estrategias basadas en la adición de especies y materiales. May et al. (1982) probó el éxito del trasplante de seis especies vegetales en el ecosistema de tundra en Colorado, Estados Unidos, ecosistema con baja productividad (Reich et al. 1997), situación análoga a la presente en los páramos neotropicales; en este estudio se encontraron porcentajes de supervivencia entre el 17 y $98 \%$ según el microambiente y la especie evaluada; sin embargo, los determinantes ambientales del éxito de la estrategias son desconocidos y se atribuye gran importancia al sistema radicular de cada una de las especies vegetales. Fagua \& González (2007) reportan un porcentaje de mortalidad de plántulas $(<5 \mathrm{~cm})$ de E. grandiflora cercano al $51 \%$ en condiciones naturales dentro del Parque Nacional Natural Chingaza, principalmente por efectos de densodependencia y estrés hídrico provocado en la época seca; por lo que en general a partir del porcentaje de supervivencia obtenido en este estudio, la reubicación de plantas jóvenes de E. grandiflora se puede considerar como una estrategia eficaz para el enriquecimiento de pastizales antrópicos de páramo.

En los meses secos se presenta la mayor mortalidad de plantas reubicadas para las tres categorías de tamaño empleadas en el área de estudio (diciembre a febrero); esto supone que los individuos de menor tamaño exhiben una menor expresión de las adaptaciones a las bajas temperaturas y el estrés hídrico, o que se presentan cambios en las estrategias adaptativas durante el ciclo de vida. Goldstein et al. (1985) encontraron que el estrés fisiológico diario no es un factor de importancia en las primeras etapas del ciclo de vida de E. timotensis, de manera que la alta mortalidad de plántulas y plantas jóvenes se debe principalmente al estrés hídrico de la época seca (Azócar \& Rada 2006). La médula de E. timotensis es capaz de compensar las pérdidas de agua por transpiración (Goldstein et al. 1984), y a pesar de que no se tenga evidencia de que la medula sea 
una adaptación que permita evadir la sequia estacional, es posible pensar que las plantas de mayor tamaño que fueron reubicadas puedan compensar de manera más eficiente el estrés hídrico que se presenta en los meses más secos ya que presentan tallos con mayor diámetro, y por consiguiente médulas más voluminosas. Este razonamiento es consistente con el hecho de que la variable que mejor predice la supervivencia luego de dos años de la reubicación es el diámetro del tallo.

A pesar que la supervivencia de las plantas jóvenes reubicadas es mayor a la encontrada en condiciones naturales, el aumento del porcentaje de supervivencia conforme aumenta el tamaño de los individuos se mantiene, lo cual es consistente con lo encontrado para la población estudiada por Fagua \& González (2007) en E. grandiflora y por Smith (1981), Monasterio \& Lamotte (1987), Estrada \& Monasterio (1988), Monasterio \& Lamotte (1989), Cavelier et al. (1992) y Silva et al. (2000) para otras especies del género. La menor mortalidad de los plantas reubicadas frente a las plantas en condiciones naturales puede deberse a la disminución de los efectos negativos de la densidad poblacional, como la presentada por E. schultzii en los páramos de Venezuela (Llambí et al. 2004), ya que al ser reubicados a distancias equidistantes puede llegar a disminuir los efectos de la densodependencia y competencia intra-específica; así mismo, el haber eliminado temporalmente la matriz de pastos exóticos pudo disminuir la competencia inter-específica generada por otras especies dominantes y aumentar la probabilidad de supervivencia.

Aunque la tasa de supervivencia de todas las categorías de tamaño se puede considerar adecuada para llevar a cabo procesos de restauración, el presentar diferencias en el porcentaje de supervivencia entre las plantas de tamaño pequeño y grande puede llegar a ser un aspecto de gran importancia para evaluar la relación costo/beneficio de la estrategia. La disponibilidad de individuos de cada uno de los tamaños no se evaluó en campo lo que imposibilita la cuantificación de los costos de la estrategia; sin embargo, a partir de las observaciones personales y el censo realizado por Fagua \& González (2007), se infiere que encontrar y extraer individuos de tamaño grande supone mayor esfuerzo, el cual debe ser cuantificado en futuras investigaciones para optimizar la estrategia.

El crecimiento de todos los individuos durante los 24 meses de estudio fue suficiente para considerarlos en la categoría de tamaño siguiente; no obstante, las plantas del género Espeletia son consideradas como de lento crecimiento (Estrada \& Monasterio 1991, Cavelier et al. 1992). La tasa de crecimiento relativo de los individuos reubicados, tanto en altura como en diámetro del tallo, es menor conforme aumenta el tamaño de la planta (Cuadro 3, Fig. $3 \mathrm{~B}$ y $3 \mathrm{C}$ ), a pesar de que los incrementos absolutos en altura no difieran entre las categorías de tamaño, y el incremento absoluto en diámetro del tallo sea mayor en la categoría grande (Cuadro 3). La mayor tasa de crecimiento relativo de las plantas de menor tamaño supone una mayor incorporación de biomasa a sus tejidos para tener incrementos similares a los presentados por las plantas de mayor tamaño.

La baja tasa de crecimiento de los individuos de mayor tamaño se compensa con la mayor tasa de renovación de hojas, lo que le confiere mayor capacidad fotosintética y una subsecuente mayor probabilidad de persistencia en áreas recolonizadas por pastos exóticos altamente competitivos. García-Orth \& Martínez-Ramos (2011) evalúan el efecto de la remoción de pastos y de la cobertura de árboles aislados sobre el desempeño de plantas de Trema micrantha trasplantadas en pastizales ubicados en la selva tropical de Chiapas, México, y al igual que Quistberg \& Stringham (2010), reportan que la remoción de los especies invasoras o altamente competitivas es el mayor determinante de la supervivencia de las plantas más no de su crecimiento. A partir de los resultados obtenidos en la alta supervivencia y el lento crecimiento de los individuos de E. grandiflora reubicados, se sugiere evaluar diferentes tratamientos enfocados a la eliminación de pastos exóticos invasores que pueden limitar la supervivencia de individuos reubicados y la eficacia de la estrategia. 
Asociados a los individuos de E. grandiflora se encuentran otras especies de plantas nativas que hacen parte de los bloques reubicados, por lo que la reubicación de individuos de E. grandiflora presenta un efecto positivo adicional al llevar consigo otras especies que pueden contribuir al enriquecimiento y la restauración del área degradada. A pesar de no ser uno de los objetivos de la investigación, se observó que los individuos de E. grandiflora de tamaño Grande presentan mayor riqueza vegetal asociada. Es de resaltar que en todos los casos se observó supervivencia de individuos de $H$. goyanesii, H. juniperinum, H. lycopodioides, $H$. mexicanum, A. nitidum y C. effusa. Hypericum spp. y A. nitidum, que corresponden a arbustos leñosos propios de las comunidades de páramo que presentan capacidad de propagarse de forma vegetativa y formar bancos de semillas (Cárdenas-Arévalo \& Vargas 2008) y C. effusa corresponde a una gramínea tipo macolla que constituye la especie que forma la matriz vegetal principal de los páramos con suelos bien drenados (Tol \& Cleef 1994), lo que las hace de gran importancia para adelantar experimentos de reubicación de individuos y ser tenidas en cuenta en los procesos de restauración ecológica del páramo.

A partir de los resultados obtenidos en este estudio se verifica el éxito de la reubicación de individuos de E. grandiflora como una de las estrategias para la restauración ecológica de los páramos andinos. Se sugiere la utilización del diámetro del tallo como variable de selección de los individuos a reubicar, ya que es la variable que mejor predice la supervivencia de las plantas jóvenes. Adicionalmente, se proporcionan valores de supervivencia y crecimiento que permitirán planear y predecir con mayor certeza programas de restauración en el páramo.

\section{AGRADECIMIENTOS}

Agradecemos al Departamento Administrativo de Ciencia Tecnología e InvestigaciónColciencias, por la financiación del proyecto "Estrategias para la restauración ecológica de los páramos andinos" dentro del cual se realizó este estudio. A la Unidad Administrativa Especial de Parques Nacionales Naturales (UAESPNN), al Parque Nacional Natural Chingaza y sus funcionarios por habernos concedido el permiso de investigación y su colaboración en la elaboración de este estudio. Al Departamento de Biología de la Universidad Nacional de Colombia, al "Grupo de Restauración Ecológica", a María Argenis Bonilla Gómez y el Grupo de investigación "Biología de Organismos Tropicales" por su formación y apoyo académico. Agradecemos a Liliana Corzo por su colaboración en la traducción de los textos; a Pilar Gómez-Ruiz, a los miembros de la Fundación Suasie, Roque, Héctor Cifuentes y la comunidad campesina aledaña al Parque por su valiosa colaboración en campo.

\section{RESUMEN}

El trasplante o reubicación de individuos ha sido ampliamente usado para la adición de especies en procesos de restauración. En este trabajo se estudió la respuesta de individuos de Espeletia grandiflora a la reubicación como estrategia de restauración ecológica; así mismo, se evaluó el efecto del tamaño de los individuos reubicados sobre la supervivencia y desarrollo. Se reubicaron individuos de tres diferentes tamaños, 5,10 y $15 \mathrm{~cm}$ de altura. Se registraron las variables supervivencia, altura de la planta, número de hojas vivas y diámetro del tallo de cada uno de los individuos, se evaluaron las posibles diferencias en cuanto a supervivencia y desarrollo. La categoría de tamaño más eficaz corresponde a la de $15 \mathrm{~cm}$ de altura, que presentó una supervivencia del $85 \%$ luego de dos años. Se sugiere el uso del diámetro del tallo como criterio de selección de los individuos a reubicar, ya que es la variable que mejor predice la supervivencia dos años luego de la reubicación. Las tasas de crecimiento relativo en altura y diámetro del tallo disminuyen conforme aumenta el tamaño de los individuos; sin embargo, el incremento absoluto en altura no presenta diferencias entre los tres tamaños evaluados. A partir de los resultados se verifica el éxito de la reubicación de plantas de E. grandiflora como estrategia de enriquecimiento en pastizales de páramos alterados.

Palabras clave: restauración ecológica, trasplantes, supervivencia, tasa de crecimiento relativo, roseta caulescente.

\section{REFERENCIAS}

Aide, T.M. \& J. Cavelier. 1994. Barriers to lowland tropical forest restoration in the Sierra Nevada de Santa Marta, Colombia. Restor. Ecol. 2: 219-229. 
Aide, T.M., J.K. Zimmerman, L. Herrera, M. Rosario \& M. Serrano. 1995. Forest recovery in abandoned tropical pastures in Puerto Rico. Forest Ecol. Manag. 77: 77-86.

Azócar, A. \& F. Rada. 2006. Relaciones hídricas, p. 99-127. In A. Azócar \& F. Rada (eds.). Ecofisiología de plantas de páramo. Instituto de Ciencias Ambientales y Ecológicas (ICAE), Facultad de Ciencias, Universidad de los Andes, Mérida, Venezuela.

Baruch, Z. \& A.P. Smith. 1979. Morphological and physiological correlates of niche breadth in two species of Espeletia (Compositae) in the Venezuelan Andes. Oecologia 38: 71-82.

Brown, S. \& A.E. Lugo. 1994. Rehabilitation of tropical lands: a key to sustaining development. Restor. Ecol. 2: $97-111$.

Buytaert, W., R. Célleri, B. De Bièvre, F. Cisneros, G. Wyseure, J. Deckers \& R. Hofstede. 2006. Human impact on the hydrology of the Andean páramos. Earth Sci. Rev. 79: 53-72.

Cárdenas-Arévalo, G. \& O. Vargas. 2008. Rasgos de historia de vida de especies en una comunidad vegetal alterada en un páramo húmedo (Parque Nacional Natural Chingaza). Caldasia 30: 245-264.

Cavelier, J., J.L. Machado, D. Valencia, J. Montoya, A. Laignelet, A. Hurtado, A. Varela \& C. Mejia. 1992. Leaf demography and growth rates of Espeletia barclayana Cuatrec. (Compositae), a caulescent rosette in a Colombian paramo. Biotropica 24: 52-63.

Díaz-Espinosa, A. \& O. Vargas. 2009. Efecto de la siembra de leguminosas herbáceas y arbustivas sobre el control en el establecimiento de la especie invasora Ulex europaeus L. (Fabaceae), en los alrededores de Chisacá (localidad de Usme. Bogotá D.C.), p. 93-130. In O. Vargas, O. León \& A. Díaz-Espinosa (eds.). Restauración ecológica en zonas invadidas por retamo espinoso y plantaciones forestales de especies exóticas. Universidad Nacional de Colombia, Bogotá, Colombia.

Ellenberg, H. 1979. Man's influence on tropical mountain ecosystems in South America: the second Tansley lecture. J. Ecol. 67: 401-416.

Estrada, C. \& M. Monasterio. 1988. Ecología poblacional de una roseta gigante, Espeletia spicata Sch. Bip. (Compositae), del páramo desértico. Ecotropicos 1: 25-39.

Estrada, C. \& M. Monasterio. 1991. Comportamiento reproductivo de una roseta gigante, Espeletia spicata Sch. Bip. (Compositae) del páramo desértico. Ecotropicos 4: 1-17.

Fagua, J.C. \& V.H. González. 2007. Growth rates, reproductive phenology, and pollination ecology of Espeletia grandiflora (Asteraceae), a giant Andean caulescent rosette. Plant Biol. 9: 127-135.
Fattorini, M. 2001. Establishment of transplants on machine-graded ski runs above timberline in the Swiss Alps. Restor. Ecol. 9: 119-126.

Fisher, R.A. 1921. Some remarks on the methods formulated in a recent article on "the quantitative analysis of plant growth”. Ann. Appl. Biol. 7: 367-372.

García-Orth, X. \& M. Martínez-Ramos. 2011. Isolated trees and grass removal improve performance of transplanted Trema micrantha (L.) Blume (Ulmaceae) saplings in tropical pastures. Restor. Ecol. 19: 24-34.

García-Varela, S. \& F. Rada. 2003. Freezing avoidance mechanisms in juveniles of giant rosette plants of the genus Espeletia. Acta Oecol. 24: 165-167.

Goldstein, G. \& F. Meinzer. 1983. Influence of insulating dead leaves and low temperatures on water balance in an Andean giant rosette plant. Plant Cell Environ. 6: 649-656

Goldstein, G., F. Meinzer \& M. Monasterio. 1984. The role of capacitance in the water balance of Andean giant rosette species. Plant Cell Environ. 7: 179-186.

Goldstein, G., F. Meinzer \& M. Monasterio. 1985. Physiological and mechanical factors in relation to sizedependent mortality in Andean giant rosette species. Oecol. Plant 6: 263-275.

Grime, J.P. 2001. Plant strategies, vegetation processes and ecosystem properties. John Wiley \& Sons, Chichester, England.

Guevara, S., S.E. Purata \& E. Maarel. 1986. The role of remnant forest trees in tropical secondary succession. Plant Ecol. 66: 77-84.

Hernández, M.L. \& R. Murcia. 1995. Estimación de la productividad primaria de Espeletia grandiflora $\mathrm{H} \&$ B y Pinus patula $\mathrm{Schl} \&$ Cham en el páramo El Granizo, Cundinamarca, Colombia, p. 503-520. In L.E. Mora-Osejo \& H. Sturm (eds.). Estudios ecológicos del páramo y del bosque altoandino cordillera Oriental de Colombia. Tomo II. Academia Colombiana de Ciencias Exactas, Físicas y Naturales, Santafé de Bogotá, Colombia.

Hofstede, R.G. 1995. Effects of burning and grazing on a Colombian paramo ecosystem. Ph.D. Thesis, Ámsterdam University, Ámsterdam, Holanda.

Hofstede, R.G., E.J.P. Chilito \& E.M. Sandovals. 1995. Vegetative structure, microclimate, and leaf growth of a paramo tussock grass species, in undisturbed, burned and grazed conditions. Plant Ecol. 119: 53-65.

Holl, K.D. 2002. Effect of shrubs on tree seedling establishment in an abandoned tropical pasture. J. Ecol. 90: 179-187.

Hunt, R. 1990. Basic growth analysis: plant growth analysis for beginners. Unwin Hyman, London, England. 
Jaimes, V. \& L. Sarmiento. 2002. Regeneración de la vegetación de páramo después de un disturbio agrícola en la cordillera oriental de Colombia. Ecotropicos 15: 61-74.

Llambí, L.D., R. Law \& A. Hodge. 2004. Temporal changes in local spatial structure of late-successional species: establishment of an Andean caulescent rosette plant. J. Ecol. 92: 122-131.

Lozano, G. \& R. Schnetter. 1976. Estudios ecológicos en el Páramo de Cruz Verde, Colombia. II. Las comunidades vegetales. Caldasia 11: 54-68.

May, D.E., P.J. Webber \& T.A. May. 1982. Success of transplanted alpine tundra plants on Niwot Ridge, Colorado. J. Appl. Ecol. 19: 965-976.

Meinzer, F. \& G. Goldstein. 1985. Some consequences of leaf pubescence in the Andean giant rosette plant Espeletia timotensis. Ecology 66: 512-520.

Meli, P. 2003. Restauración ecológica de bosques tropicales. Veinte años de investigación académica. Interciencia 28: 581-589.

Molinillo, M. 1992. Pastoreo en ecosistemas de páramo: estrategias culturales e impacto sobre la vegetación en la cordillera de Mérida, Venezuela. Tesis de Maestría, Universidad de Los Andes, Mérida, Venezuela.

Molinillo, M. \& M. Monasterio. 1997. Pastoralism in paramo environments: practices, forage, and impact on vegetation in the cordillera of Merida, Venezuela. Mt. Res. Dev. 17: 197-211.

Molinillo, M. \& M. Monasterio. 2002. Patrones de vegetación y pastoreo en ambientes de páramo. Ecotropicos 15: 19-34.

Monasterio, M. \& M. Lamotte. 1987. La production d'une population d' Espeletia timotensis dans un écosystème de haute montagne tropicale (Andes du Venezuela). C.R. Acad. Sci. Paris. 305: 687-690.

Monasterio, M. \& M. Lamotte. 1989. Les populations d' Espeletia timotensis dans le páramo désertique des Andes du Venezuela. Revue d'écologie 44: 301-327.

Monasterio, M. \& L. Sarmiento. 1991. Adaptive radiation of Espeletia in the cold andean tropics. Trends Ecol. Evol. 6: 387-391.

Nepstad, D.C., C. Uhl, C.A. Pereira \& J.M.C. da Silva. 1996. A comparative study of tree establishment in abandoned pasture and mature forest of eastern Amazonia. Oikos 76: 25-39.

Orozco, N. 2007. Crecimiento y desarrollo de cuatro especies nativas en el corredor ripario potrerizado del río Chisacá, sector Capilla del Hato, localidad de Usme, Bogotá D.C., p. 382-401. In O. Vargas (ed.). Restauración ecológica del bosque altoandino. Estudios diagnósticos y experimentales en los alrededores del embalse de Chisacá, localidad de Usme, Bogotá D.C. Universidad Nacional de Colombia, Bogotá, Colombia.
Parrotta, J.A. \& O.H. Knowles. 1999. Restoration of tropical moist forests on bauxite-mined lands in the Brazilian Amazon. Restor. Ecol. 7: 103-116.

Quistberg, S.E. \& T.K. Stringham. 2010. Sedge transplant survival in a reconstructed channel: influences of planting location, erosion, and invasive species. Restor. Ecol. 18: 401-408.

Rangel-Ch., J.O. \& H. Sturm. 1995. Consideraciones sobre la vegetación, la productividad neta y la artropofauna asociada en regiones paramunas de la Cordillera Oriental, p. 47-70. In L.E. Mora-Osejo \& H. Sturm (eds.). Estudios ecológicos del páramo y del bosque altoandino cordillera Oriental de Colombia. Tomo I. Academia Colombiana de Ciencias Exactas, Físicas y Naturales, Santafé de Bogotá, Colombia.

Rangel-Ch., O. \& C. Ariza-N. 2000. Nuevos tratamientos sobre la vegetación del páramo, p. 720-784. In O. Rangel-Ch (ed.). Colombia Diversidad Biótica III. La región de vida paramuna. Universidad Nacional de Colombia, Bogotá, Colombia.

Reich, P.B., M.B. Walters \& D.S. Ellsworth. 1997. From tropics to tundra: Global convergence in plant functioning. Proc. Natl. Acad. Sci. Unit. States Am. 94: 13730-13734.

Sarmiento, F.O. 1997. Arrested succession in pastures hinders regeneration of Tropandean forests and shreds mountain landscapes. Environ. Conservat. 24:14-23.

Sarmiento, F.O. 2000. Breaking mountain paradigms: ecological effects on human impacts in man-aged tropandean landscapes. Ambio 29: 423-431.

Sarmiento, L., L. Llambí, A. Escalona \& N. Marquez. 2003. Vegetation patterns, regeneration rates and divergence in an old-field succession of the high tropical Andes. Plant Ecol. 166: 145-156.

Sendoya, S.F. \& M.A. Bonilla. 2005. La necromasa de Espeletia grandiflora como hábitat para la artropofauna del páramo, p. 197-226. In M.A. Bonilla (ed.). Estrategias adaptativas de plantas del páramo y del bosque altoandino en la cordillera oriental de Colombia. Universidad Nacional de Colombia, Bogotá, Colombia.

Silva, J.F., M.C. Trevisan, C.A. Estrada \& M. Monasterio. 2000. Comparative demography of two giant caulescent rosettes (Espeletia timotensis and E. spicata) from the high tropical Andes. Global Ecol. Biogeogr. 9: 403-413.

Slocum, M.G. 2000. Logs and fern patches as recruitment sites in a tropical pasture. Restor. Ecol. 8: 408-413.

Smith, A.P. 1974. Bud temperature in relation to nyctinastic leaf movement in an Andean giant rosette plant. Biotropica 6: 263-266.

Smith, A.P. 1979. Function of dead leaves in Espeletia schultzii (Compositae), and Andean caulescent rosette species. Biotropica 11: 43-47. 
Smith, A.P. 1981. Growth and population dynamics of Espeletia (Compositae) in the Venezuelan Andes. Smithsonian Contributions to Botany no. 48. Smithsonian Institution, Washington, USA

Sturm, H. \& O. Rangel-Ch. 1985. Ecología de los páramos Andinos. Una visión preliminar integrada. Biblioteca José Jerónimo Triana, Instituto de Ciencias Naturales No. 9. Universidad Nacional de Colombia, Bogotá, Colombia.

Suarez, E. 2008. Integridad ecológica frente a salud ecosistémica: reflexiones sobre enfoques de conservación en ecosistemas de páramo, p. 39-51. In P. Mena Vásconez, M. Morales, P. Ortiz, G. Ramón, S. Rivadeneira, E. Suarez, J.F. Terán \& C. Velásquez (eds.). Gente y Ambiente de páramo: Realidades y Perspectivas en el Ecuador. Ecociencia-Abya Yala. Quito, Ecuador (también disponible en línea: http://www.ecociencia. org/archivos/gente_paramo-091128.pdf).

Tol, G.J. \& A.M. Cleef. 1994. Above-ground biomass structure of a Chusquea tessellata bamboo páramo, Chingaza National Park, cordillera oriental, Colombia. Plant Ecol. 115: 29-39.

Trujillo-Ortiz, L. 2007. Evaluación de la regeneración natural y sobrevivencia de especies nativas en parcelas experimentales en potreros, p. 402-424. In O. Vargas (ed.). Restauración ecológica del bosque altoandino. Estudios diagnósticos y experimentales en los alrededores del embalse de Chisacá, localidad de Usme, Bogotá D.C. Universidad Nacional de Colombia, Bogotá, Colombia.

Urbanska, K.M. 1994. Ecological restoration above the timberline: demographic monitoring of whole trial plots in the Swiss Alps. Bot. Helv. 104: 141-156.

Vázquez-Yanes, C. \& A. Orozco-Segovia. 1993. Patterns of seed longevity and germination in the tropical rainforest. Annu. Rev. Ecol. Syst. 24: 69-87.

Vargas, O., J. Premauer \& CdlA. Cárdenas. 2002. Efecto del pastoreo sobre la estructura de la vegetación en un páramo húmedo de Colombia. Ecotropicos 15: 35-50.

Vargas, O. \& P. Pedraza. 2004. Parque Nacional Natural Chingaza. Universidad Nacional de Colombia, Bogotá, Colombia.

Verweij, P.A. \& K. Kok. 1992. Effects of fire and grazing on Espeletia hartwegiana populations, p. 215-229. In H. Balslev \& J.L. Luteyn (eds.). Páramo. An Andean ecosystem under human influence. Academic, London, England.

Wallin, L., B.M. Svensson \& M. Lönn. 2009. Artificial dispersal as a restoration tool in meadows: sowing or planting? Restor. Ecol. 17: 270-279.

Zimmerman, J.K., J.B. Pascarella \& T.M. Aide. 2000. Barriers to forest regeneration in an abandoned pasture in Puerto Rico. Restor. Ecol. 8: 350-360. 